\title{
Review Paper: Polyphenolic Antioxidants and Neuronal Regeneration
}

\author{
Amin Ataie ${ }^{1}$, Mohammad Shadifar ${ }^{2}$, Ramin $_{\text {Ataee }^{3 *}}$ \\ 1. Cellular and Molecular Research Center, Babol University of Medical Sciences, Babol, Iran. \\ 2. Amol Center, Pasteur Institute of Iran, Amol, Iran. \\ 3. Department of Pharmacology and Toxicology, Pharmaceutical Sciences Research Center, Mazandaran University of Medical Sciences, Sari, Iran.
}

Citation: Ataie, A., Shadifar, M., \& Ataee, R. (2016). Polyphenolic antioxidants and neuronal regeneration. Basic and Clinical Neuroscience, 7(2), 81-90. http://dx.doi.org/10.15412/J.BCN.03070201

http://dx.doi.org/10.15412/J.BCN.03070201

Article info:

Received: 05 May 2015

First Revision: 17 June 2015

Accepted: 13 August 2015

Key Words:

Curcumin, Alzheimer, Resveratrol, Oxidative stress

\begin{abstract}
A B S T R A C T
Many studies indicate that oxidative stress is involved in the pathophysiology of neurodegenerative diseases. Oxidative stress can induce neuronal damages, modulate intracellular signaling and ultimately leads to neuronal death by apoptosis or necrosis. To review antioxidants preventive effects on oxidative stress and neurodegenerative diseases we accumulated data from international medical journals and academic informations' sites. According to many studies, antioxidants could reduce toxic neuronal damages and many studies confirmed the efficacy of polyphenol antioxidants in fruits and vegetables to reduce neuronal death and to diminish oxidative stress. This systematic review showed the antioxidant activities of phytochemicals which play as natural neuroprotectives with low adverse effects against some neurodegenerative diseases as Parkinson or Alzheimer diseases.
\end{abstract}

\section{Introduction}

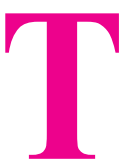

here are a wide variety of neurodegenerative diseases such as Alzheimer disease, Parkinson disease, multiple sclerosis, Huntington disease and the like. Their cases are sporadic and therefore, finding the etiology of these diseases and preventing them are challenging. Oxidative stress is recognized as a common factor in many neurodegenerative diseases and age-related degenerative processes. The pathogenesis of neurodegenerative diseases is multifactorial with a complex combination of genetic components and environmental factors. The environmental or sporadic form represents the majority of these cases. Toxic reactions, including inflammation, glutamatergic toxicity, dysfunction of mitochondrial activity and ubiquitin/proteasome system, activation of apoptosis pathways, elevation of iron and nitric oxide, and alteration of the homeostasis of antioxidants/oxidation are involved in the pathogenesis of neurodegenerative diseases (Ataie, Sabetkasei, Haghparast, Moghaddam, \& Kazemi-Nejad, 2010).

There is evidence indicating that different reactive oxygen species (ROS), for example, superoxide, hydrogen peroxide, as well as hydroxyl and peroxyl radicals, are produced in the cells under normal and pathological conditions (Sun \& Cheng, 1999). When the rate of ROS generation exceeds the capacity of antioxidant defense, oxidative damage to DNA, proteins, and lipids will oc-

\section{* Corresponding Author:}

Ramin Ataee, PhD

Address: Department of Pharmacology, Faculty of Pharmacy, Mazandaran University of Medical Sciences, Sari, Iran.

Tel:+98 (911) 3232804

E-mail: raminataee1349@gmail.com 
cur (Sun \& Cheng, 1999). In the central nervous system (CNS), oxidative stress is implicated in mechanisms leading to neuronal cell injury in various pathological states. Recently, the term "nitrosative stress" has been used to indicate cellular damage elicited by reactive nitrogen species (RNS), which include nitric oxide (NO) and its derivatives such as peroxynitrite and nitroxyl anion (Kelsey, Wilkins, \& Linseman, 2010). Together, oxidative and nitrosative stresses are implicated in the pathology of many neurodegenerative disorders, including Alzheimer disease (AD), Parkinson disease (PD), Huntington disease (HD), amyotrophic lateral sclerosis (ALS), and stroke (Kelsey, Wilkins, \& Linseman, 2010).

\subsection{Brain and oxidative stress}

The brain is particularly vulnerable to oxidative damage because it utilizes a large amount of oxygen for energy and has relatively low antioxidant defense enzymes, especially during aging. In addition, membranes in brain cells contain abnormally high proportions of polyunsaturated fatty acids (PUFAs) (Wu, 2005). Of the different types of cells in the brain, neurons are especially vulnerable to toxic compounds, and sensitive to damage by ischemia/stroke, seizure, and other excitotoxic injury. Oxidative damage to lipids (lipid peroxidation) is associated with progressive loss of membrane integrity, reduction of mitochondrial membrane potential, and increase in plasma membrane permeability to $\mathrm{Ca}^{2+}$. Oxidative damage to proteins leads to the formation of carbonyl and nitrosylated derivatives (Kelsey, Wilkins, \& Linseman, 2010). Furthermore, ROS damage to DNA results in nuclear condensation and altered gene expression (Kelsey, Wilkins, \& Linseman, 2010). Therefore, oxidative stress is an important risk factor for neurodegeneration. In recent years, extensive effort has been devoted to develop novel strategies for overcoming different types of brain damages (Sun, Wang, Simonyi, \& Sun, G. Y, 2008).

\subsection{Natural antioxidant}

Many antioxidant compounds derived from natural products (nutraceuticals) have demonstrated neuroprotective activity in either in vitro or in vivo models of neuronal cell death. These natural antioxidants are as follows: (1) Flavonoid polyphenols like epigallocatechin 3-gallate (EGCG) from green tea and quercetin from apple; (2) Non-flavonoid polyphenols such as curcumin from turmeric and resveratrol from grape; (3) Phenolic acids or phenolic diterpenes such as rosmarinic acid or carnosic acid, respectively, both from rosemary; and (4)
Organosulfur compounds, including isothiocyanate and L-sulforaphane, from broccoli and the thiosulfinate (allicin), from garlic (Kelsey, Wilkins, \& Linseman, 2010). All of these compounds are antioxidants. They directly scavenge free radicals or indirectly increase endogenous cellular antioxidant defenses, for example, via activation of the nuclear factor erythroid-derived 2-related factor 2 (Nrf2) transcription factor pathways (Kelsey, Wilkins, \& Linseman, 2010). Other mechanisms of action of these compounds are modulation of signal transduction cascades or effect on gene expression (Kelsey, Wilkins, \& Linseman, 2010).

A common method of determining intrinsic free radical scavenging activity is to use a cell free assay system with the radical 2, 2-diphenyl-1-picryhydrazyl (DPPH) (Kelsey, Wilkins, \& Linseman, 2010). Resveratrol, carnosic acid, and rosmarinic acid have been shown to be effective scavengers of DPPH radicals (Kelsey, Wilkins, \& Linseman, 2010). In contrast, allicin has been found to be a poor scavenger of peroxyl radicals while another garlic compound, 2-propenesulphenic acid, is a good scavenger of these radicals. Additionally, EGCG has been shown to scavenge a wide variety of free radicals, including superoxide, hydroxyl radical, hydrogen peroxide, and nitric oxide (Kelsey, Wilkins, \& Linseman, 2010). The intrinsic free radical scavenging activities of these nutraceuticals antioxidants suggest that they may have potential utility in mitigating neuronal oxidative stress and neurodegeneration (Kelsey, Wilkins, \& Linseman, 2010).

\subsection{Neurodegenerative disorders}

Histopathologic alterations in Alzheimer disease are characterized by neurofibrillary tangles and senile plaques. Amyloid- $\beta$ peptide is the main component of senile plaques and has a causal role in the development and progress of Alzheimer disease (Hardy \& Higgins, 1992). This peptide is toxic to neurons through different mechanisms, including apoptosis, mitochondrial dysfunction, or activation of

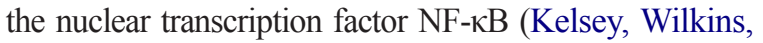
\& Linseman, 2010). Moreover, the aggregation and toxicity of the amyloid- $\beta$ peptide involve transition of metals (Atwood et al., 1998), formation of hydrogen peroxide (Behl, Davis, Lesley, \& Schubert, 1994), accumulation of reactive oxygen species and oxidative stress (Behl, Davis, Lesley, \& Schubert, 1994) that leads to neuronal death. Accordingly, there is considerable evidence that oxidative stress is an early and critical event in the pathogenesis of Alzheimer disease (Nunomura et al., 1999).

Studies revealed that the levels of lipid oxidation products (e.g. malondialdehyde, 4-hydroxynonenal, and isoprostane), 
protein oxidation (e.g. protein carbonyl, nitro tyrosine), and DNA oxidation are elevated in the brain of patients with Alzheimer disease (Christen, 2000). Moreover, the level of lipid oxidation in hippocampus and frontal cortex of patients with Alzheimer disease is dependent on the apolipoprotein $\mathrm{E}$ genotype (Ramassamy \& Poirier, 2001). Protein carbonyls are present in both tangles- and non-tangles-bearing neurons (Smith \& Luo, 2003) and hippocampus neurons demonstrate intense cytoplasmic staining with 8-hydro-2-deoxyguanosine (Nunomura et al., 1999). Controlled clinical trial was conducted with the lipophilic antioxidant $\alpha$-tocopherol. High doses of $\alpha$-tocopherol are prescribed for patients with Alzheimer disease; It has some beneficial effect on the rate of deterioration of cognitive functions (Sano et al., 1997). On the other hand, epidemiological studies indicate that dietary habits and antioxidants from diet can influence the incidence of neurodegenerative disorders such as Alzheimer and Parkinson diseases (Morris et al., 2002).

There is evidence that medications or vitamins that increase the levels of brain catecholamine and protect against oxidative damage may reduce the neuronal damage and slow the progression of Alzheimer disease (Sano et al., 1997). Sano et al. showed that taking vitamin $\mathrm{E}$ from food, but not other antioxidants, may be associated with a reduced risk of $\mathrm{AD}$. Unexpectedly, this association was observed only among individuals without the APOE E4 allele. Antioxidant nutrients, including vitamin $\mathrm{E}$, vitamin $\mathrm{C}$, and beta carotene, are among the body's natural defense mechanisms against oxidative stress. The antioxidant nutrients have been shown (through animal and laboratory studies of brain tissue) to decrease lipid peroxidation (Abd el-Fattah, al-Yousef, alBekairi, \& al-Sawaf, 1998) and the oxidation of proteins; inhibit the production of reactive oxygen species; prevent mitochondrial dysfunction and DNA fragmentation (Tagami et al., 1998); and reduce neurotoxicity, apoptosis, and neuronal death (Mason et al., 1999).

Few studies have examined the relation between dietary intake of antioxidant nutrients and the development of AD. Two prospective studies (Masaki et al., 2000) that reported on the association of vitamin $\mathrm{E}$ and vitamin $\mathrm{C}$ supplement use and $\mathrm{AD}$ yielded conflicting results, but both had limited power to test the hypothesis, and neither had dietary information. Sano et al. reported on the association of AD incident with intake of antioxidant nutrients from foods and supplements in a large community study (Sano et al., 1997).

Furthermore, lesions are present in the brains of patients with $\mathrm{AD}$ that are typically associated with attacks of free radicals (e.g. damage to DNA, protein oxidation, lipid peroxidation, and advanced glycosylation end products), and metals (e.g. iron, copper, zinc, and aluminum) are pres- ent that have catalytic activity and produce free radicals. In the presence of free radicals, $\beta$-Amyloid peptide aggregates and produces more free radicals; $\beta$-amyloid toxicity is eliminated by free radical scavengers. Apolipoprotein $\mathrm{E}$ is subject to attacks by free radicals, and apolipoprotein E peroxidation has been correlated with $\mathrm{AD}$ (Christen, 2000). In contrast, apolipoprotein $E$ can act as a free radical scavenger, which is isoform dependent. $\mathrm{AD}$ has been linked to mitochondrial anomalies affecting cytochrome oxidase, and these anomalies may contribute to the abnormal production of free radicals. Finally, many free radical scavengers (e.g. vitamin E, selegiline, and Ginkgo biloba extract EGb 761) have shown promising results in relation to $\mathrm{AD}$, as has desferrioxamine, an iron-chelating agent, and anti-inflammatory drugs and estrogens, which also have an antioxidant effects (Christen, 2000).

A small number of studies have yielded inconsistent results with varying lengths of follow-up. In Rotterdam Study, It is found that higher dietary intake of vitamins $\mathrm{E}$ and $\mathrm{C}$ was associated with a lower risk of dementia and $\mathrm{AD}$ over 6 years of follow-up. Still, substantial evidence indicates that earlier exposures are important for predicting dementia risk in later life (Launer, 2005), and specific evidence indicates that antioxidants may influence early stages of dementia development (Berr, Balansard, Arnaud, Roussel, \& Alpérovitch, 2000). Therefore, the association of dietary vitamin E, vita$\min \mathrm{C}$, beta carotene, and flavonoids with long-term risk of dementia was evaluated based on ten years of follow-uptaking advantage of both longer follow-up and substantially more dementia cases (Devore et al., 2010).

\section{Materials \& Methods}

\subsection{Polyphenol antioxidants}

Flavonoids can be divided into various classes on the basis of their molecular structure such as anthocyanins and anthoxanthins (Figure 1). The latter group is divided into flavonoids, flavons, flavanols, flavonones, and isoflavones with catechins being the best described flavanols (Figure 1). Although, the data available on phenolic bioavailability are still limited, it has been shown that the eating the foods or beverages which are rich in polyphenols can increase the antioxidant levels in the body (Morel et al., 1993). These results suggest that the consumption of polyphenolic compounds could have beneficial effect on oxidative-induced damages. There are many chemical classes of nutraceuticals found in all sorts of foods. Some nutraceuticals are well known, like epigallocatechin 3-gallate (EGCG) from green tea and resveratrol from grapes, while others are largely unknown to the consumer. The chemical structures of the natural compounds 
reviewed here are shown in figure 2. Although these compounds differ structurally, each of them has been shown to have neuroprotective and antioxidant properties.

A common method of determining intrinsic free radical scavenging activity is to use a cell free assay system with the radical 2, 2-diphenyl-1-picryhydrazyl (DPPH) (Kelsey, Wilkins, \& Linseman, 2010). Resveratrol (Shang et al., 2009), carnosic acid (Miura, Kikuzaki, \& Nakatani, 2002), and rosmarinic acid (Alamed, Chaiyasit, McClements, \& Decker, 2009) have been shown to be effective scavengers of DPPH radicals. In contrast, allicin has been found to be a poor scavenger of peroxyl radicals while another garlic compound, 2-propenesulphenic acid, is a good scavenger (Galano \& Marquez, 2009). Additionally, EGCG has been shown to scavenge a wide variety of free radicals, including superoxide, hydroxyl radical, hydrogen peroxide, and nitric oxide (Paquay et al., 2000; Qi, 2010). The intrinsic free radical scavenging activities of these nutraceutical antioxidants suggest that they may have potential use in the treatment of neuronal oxidative stress and neurodegeneration (Kelsey, Wilkins, \& Linseman, 2010).

\subsubsection{Epigallocatechin}

Epigallocatechin (EGCG) is a flavonoid polyphenol and the main antioxidant compound in green tea, which shows neuroprotective effects. For example, mitochondrial function is improved by antioxidative action of EGCG. Moreover, EGCG ameliorates lipid infusion-mediated insulin resistance, which is associated with increased expression of antioxidant enzymes including superoxide dismutase (SOD) and glutathione peroxidase by EGCG in vivo (Li et al., 2011). Schroeder et al. (2009) has shown that EGCG selectively protects cultured rat cerebellar granule neurons (CGNs) from oxidative stress (Schroeder et al., 2009). Some studies have shown that EGCG significantly diminish oxidative stress and neuronal death induced by hydrogen peroxide in motor neurons (Koh et al., 2004); likewise EGCG protects SH-SY5Y human neuroblastoma cells from amyloid precursor protein (APP), 3-hydroxykynurenine, or 6-hydroxydopamine (6-OHDA) toxicity (Levites, Amit, Youdim, \& Mandel, 2002).

In addition to the neuroprotective effects of EGCG observed in vitro, this nutraceutical antioxidant also preserves neuronal survival and function in several in vivo models of neurodegeneration. For example, oral administration of EGCG protects mice from the dopaminergic toxicity caused by the Parkinson neurotoxin, 1-methyl4-phenyl-1, 2, 3, 6-tetrahydropyridine (MPTP). EGCG treatment prevents the MPTP-induced loss of dopamine neurons from the substantia nigra pars compacta and preserves striatal dopamine levels in mice (Levites, Weinreb, Maor, Youdim, \& Mandel, S, 2001). Oral administration of EGCG to transgenic mice expressing a human G93A mutant SOD1 (Cu, Zn-superoxide dismutase) gene significantly delays symptoms onset and moderately extends life span when compared to vehicle treated mice (Koh et al., 2004). EGCG also reduces photoreceptor degeneration and improves motor function in a Drosophila model of Huntington disease (Ehrnhoefer et al., 2006). The catechol-like structure of catechins may competitively inhibit the uptake by the presynaptic or vesicular transporters of the metabolite product of MPTP, 1-methyl-4-phenylpyridinium ion (MPP+) having also a catechol-like structure (Pan, Fei, Zhou, Jankovic, \& Le, 2003). This competition could protect dopaminergic neurons against the MPTP/ $\mathrm{MPP}+-$ induced injury (Pan, Fei, Zhou, Jankovic, \& Le, 2003).

Tea polyphenols have been found to be potent scavengers of singlet oxygen, superoxide anions, hydroxyl radicals, and peroxyl radicals (Morel et al., 1993). Although there is no significant data about effectiveness of tea in Alzheimer disease treatment, there are several in vitro studies indicating that green tea extract could protect neurons from the amyloid $\beta$-induced damages (Bastianetto, Yao, Papadopoulos, \& Quirion, 2006). Over the past decade, intense research has been focused to investigate the processes of the amyloid precursor protein (APP) proteolysis and amyloid- $\beta$ metabolism as possible targets for the therapy of Alzheimer disease. APP can be processed by two pathways: 1) A non-amyloidogenic pathways which involve cleavage of APP to soluble APP (sAPP) by $\alpha$-secretase activity; and 2) Formation of the amyloidogenic $\beta$ peptides by the $\beta$-secretase and $\gamma$-secretase (Ramassamy \& Poirier, 2001).

\subsubsection{Resveratrol}

Resveratrol (trans-3,4',5-trihydroxystilbene) is the main nonflavonoid polyphenol found in grapes and red wine (Figure 2). It has been shown to possess many biological and pharmacological properties, including antioxidant, anti-inflammatory, antimutagenic, and anticarcinogenic effects (Jang et al., 1997). Resveratrol (Figure 2 ) is a polyphenolic antioxidant found in many kinds of grapes and is known mostly for its cardiovascular benefits (Bertelli \& Das, 2009). However, resveratrol also shows significant neuroprotective activity in vitro and in vivo. In some in vitro studies, resveratrol protects hippocampus from oxygen-glucose deprivation (Zamin et al., 2006), also embryonic rat mesencephalic cultures from tert-butyl hydroperoxide (Karlsson, Emgard, Brundin, \& 
Burkitt, 2000), and CGNs from MPP(+)-induced toxicity (Alvira et al., 2007). In vivo, resveratrol significantly attenuates hippocampal neurodegeneration and learning impairment in the inducible $\mathrm{p} 25$ transgenic mouse model of AD and tauopathy (Kim et al., 2007). Moreover, resveratrol reduces oxidative damage and preserves striatal dopamine in the 6-OHDA rat model of PD (Khan et al., 2010). For instance, in PC12 cells, resveratrol, in $10 \mu \mathrm{M}$ concentration has been shown to protect against the amyloid- $\beta$ peptide-induced toxicity.

In agreement with its antioxidant activity, the amyloid- $\beta$ peptide-induced intracellular accumulation of reactive oxygen species was attenuated by resveratrol. Reactive oxygen species and amyloid- $\beta$ peptide can also induce cell death via apoptosis in many cell types. Such effect was also blocked by resveratrol. It can prevent the cleavage of poly (ADP-ribose) polymerase (PARP), a substrate of active caspase-3, and decrease in the ratio of Bcl-x/Bax or activation of JNK. Resveratrol can protect hippocampal primary neurons against the toxicity of amyloid $\beta$ peptide. Also a treatment with resveratrol, administrated 2 hours after the amyloid- $\beta$ peptide (25-35) $(20 \mu \mathrm{M})$, significantly diminished the amyloid- $\beta$ peptideinduced cell death relative to its dosage. Miloso et al. have shown that resveratrol can induce the activation of the MAP kinases, ERK1, and ERK2. Besides these signaling pathways (Miloso, Bertelli, Nicolini, \& Tredici, 1999) resveratrol can induce the expression of the transcription factor early growth response (EGR1) (Della et al., 2002). EGR1 transcription factor could regulate some aspects of synaptic plasticity-related to learning and memory (Li et al., 2005). Recently, in two different APP695-transfected cell lines (HEK293 and N2A), Marambaud et al. showed that resveratrol $(20-40 \mu \mathrm{M})$ could markedly reduce the secretion of the amyloid- $\beta$ peptide (1-40) (Marambaud, Zhao, \& Davies, 2005). This effect of resveratrol occurred without directly affecting $\beta$-secretases and $\gamma$-secretases since resveratrol has no effect on these enzymes (Marambaud, Zhao, \& Davies, 2005).

\subsubsection{Curcumin}

The yellow curry spice, curcumin, a non-flavonoid polyphenol shows anti-inflammatory and antioxidant activities (Figure 3). Epidemiological studies have raised the possibility that this molecule used by Asian Indian population is involved for the significantly lower prevalence of Alzheimer disease in India compared to the United States (4.4 fold) (Ganguli et al. 2000). In Neuro2a mouse neuroblastoma cells infected with Japanese encephalitis virus, curcumin enhances cell viability by decreasing ROS and inhibiting pro-apoptotic signals (Dutta, Ghosh, \& Basu, 2009). In vivo, curcumin protects rats from focal cerebral ischemia induced by middle cerebral artery occlusion (Yang, Zhang, Fan, \& Liu, 2009).

In addition, curcumin is neuroprotective against the MPTP-induced neurodegeneration of the nigrostriatal tract in mice and was shown to prevent glutathione depletion and lipid peroxidation induced by this toxin. Furthermore, curcumin displays an additive protective effect to that of catalase and SOD activities in the striatum and midbrain of MPTP-treated mice (Rajeswari, 2006). We showed that curcumin could protect rat hippocampus cells against homocysteine (Hcy) neurotoxicity (Ataie et al., 2010). We indicated that Hcy was neurotoxic for rats and biochemical results revealed that 5 days after Hcy intrahippocampal injection in rat brain, the levels of MDA and superoxide anion were significantly increased in the hippocampi in comparison to the vehicle and control groups (Ataie et al., 2010). It was observed that Hcy overdose can be lethal for rats and some morbidity and mortality and Parkinson like behaviors (tremor, rotation) were observed after administration of $0.4-\mu \mathrm{mol} / \mu \mathrm{L}$ or higher concentrations of Hcy.

Furthermore, our results indicated that Hcy $(0.2-\mu \mathrm{mol} / \mu \mathrm{L})$ could diminish passive avoidance learning behavior significantly, and decreased latency time in the retention test. The Hcy group had significantly lower latency time than other groups, indicating that Hcy could diminish long term as well as short term memory in rats (Ataie et al., 2010).

\section{Results}

Results of our study indicated that curcumin treatment in both low and high doses ( 5 and $50 \mathrm{mg} / \mathrm{kg}$ ) inhibited lipid peroxidation significantly and decreased MDA and SOA levels in the Hcy treated hippocampi (Ataie et al., 2010). Nonetheless, curcumin also improved Hcy-mediated memory deficits and increased latency times in the first and second passive avoidance tests (Ataie et al., 2010). Also, histopathological analysis showed that Hcy reduced cell density in some parts of hippocampus. Cell density in dentate gyrus of the dorsal hippocampus was decreased in the Hcy group in comparison to the vehicle group.

However, this deficit was significantly improved by treatment with curcumin (Ataie et al., 2010). In the other study, we revealed that curcumin could protect substantia nigra cells in rat brain against Hcy neurotoxicity. The findings of our study revealed that curcumin at a dose of $50 \mathrm{mg} / \mathrm{kg}$ was able to significantly reverse be- 


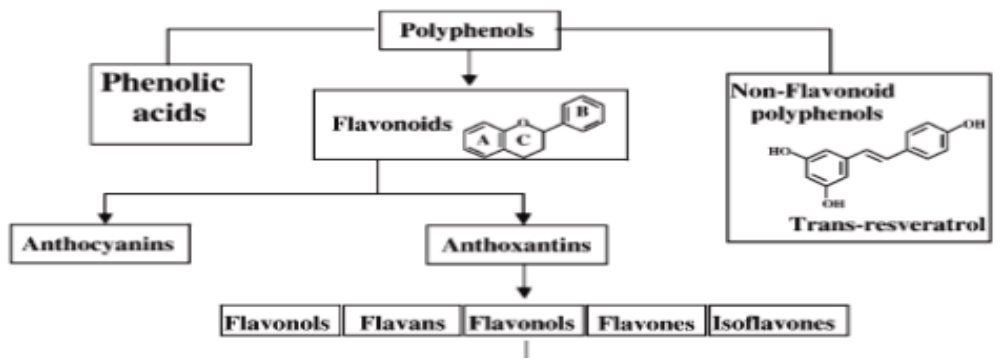

NEUR:SCIENCE

Figure 1. Polyphenol structure includes phenolic acid, flavonoid, and non-flavonoid polyphenols.

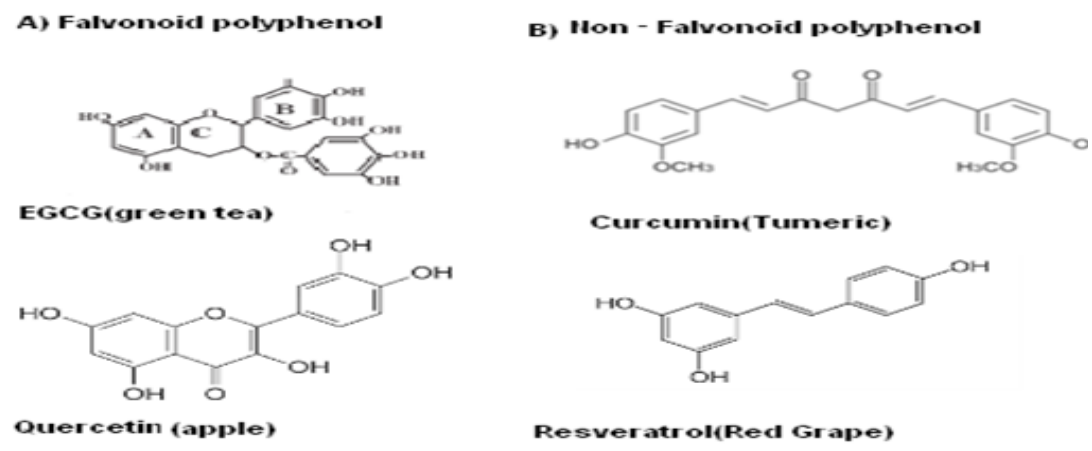

Figure 2. Structure of flavonoid and non-flavonoid polyphenols.

NEUR:SCIENCE

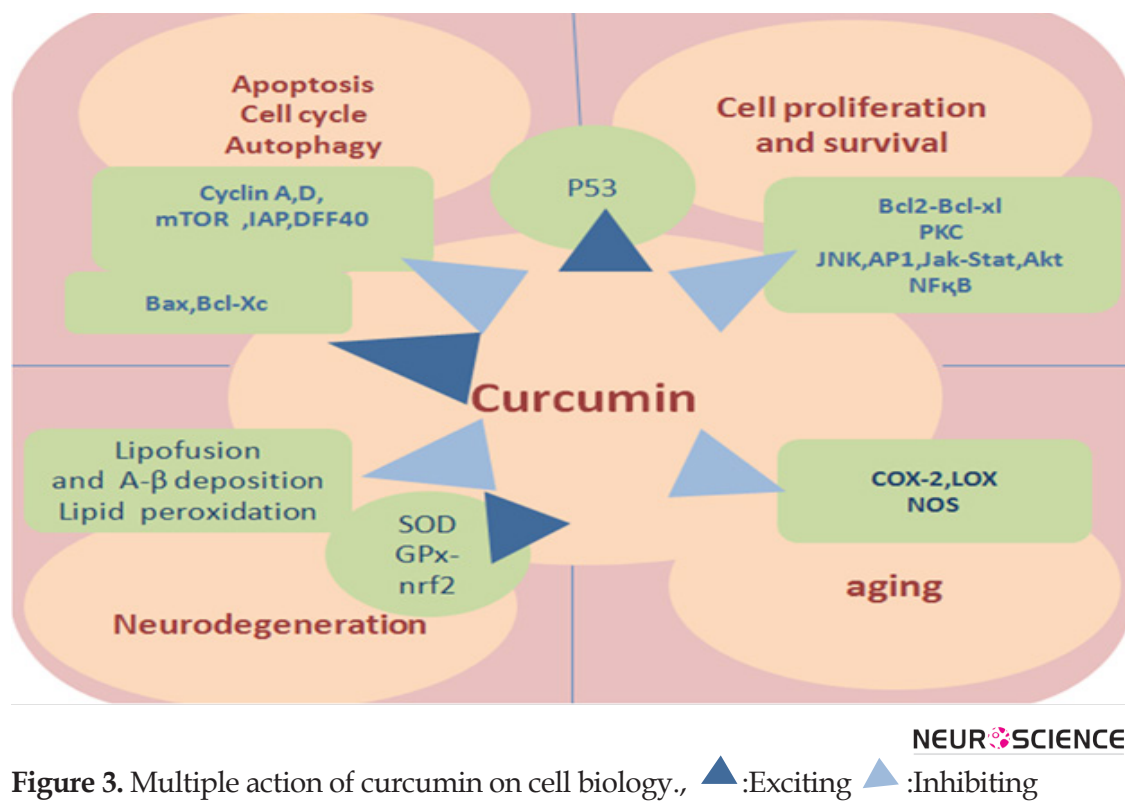

havioral and biochemical changes caused by exposure of homocysteine in rats. In the other study, when transgenic APPsw mice received curcumin in their diet for 6 months, indexes of inflammation and oxidative damages suppressed (Lim et al., 2001). Also a reduction of IL-1 $\beta$, a proinflammatory cytokine, and a decrease in oxidized proteins were seen. Furthermore, the levels of insoluble, soluble amyloid- $\beta$ peptide, and plaque burden significantly decreased. These effects were associated with the 
suppression of microgliosis and a marked reduction of glial fibrillary proteins GFAP, an astrogliosis marker.

These findings could be explained by curcumin property to inhibit the amyloid- $\beta$ fibril formation (Kim et al., 2005). The protective effect of curcumin against the amyloid- $\beta$ peptide was also observed on $\mathrm{PC} 12$ cells in culture (Kim et al., 2005). Also, curcumin could bind to the redox-active iron and copper (Baum \& Ng, 2004). Thus, it may exert protective effect against oxidative damages initiated by divalent metals or suppress inflammatory damage by preventing metal induction of NF- $\mathrm{KB}$. On the other hand, curcumin is a highly lipophilic compound and cross the blood brain barrier where it can bind to plaques and prevent the amyloid- $\beta$ peptide aggregation (Baum \& Ng, 2004).

\section{Discussion}

Polyphenol antioxidants have properties to treat neurodegenerative diseases. Many of these natural antioxidants are not only active scavengers of free radicals but also modulators of prosurvival or proapoptotic signaling pathways (Kelsey, Wilkins, \& Linseman, 2010).

Also, these compounds may have a greater potential than synthetic drugs with only one mechanism of action. Polyphenols reduce oxidative stress and promote neuronal survival signals in many models (in vitro and in vivo) of neuronal injury and neurodegenerative disease. Although individual neurodegenerative diseases demonstrate in distinct neuronal cell types, oxidative stress and suppression of neuronal survival signals are common in many pathological conditions and mark many targets for treatment. Therefore, the effective treatment for neurodegenerative diseases is very hard to find. A lot of funding and research have been allocated for treatment of $\mathrm{AD}$ and $\mathrm{PD}$, yet even these diseases have only calmative therapies available and none that significantly slow or stop the underlying pathology of the disease. Polyphenol antioxidants may be the best choice for these patients in the short term since they are subject to fewer regulations than traditional pharmaceuticals and therefore, could be made available to patients much more rapidly.

Finally, another evidence for the enormous potential of polyphenols antioxidants as novel therapeutics for neurodegeneration includes the recent initiation of several clinical trials with these compounds. EGCG (green tea) is currently being tested in phase II trials for PD and early stage AD (Charite University, Berlin, Germany). Similarly, resveratrol is being tested in a phase II trial to improve memory performance in the elderly.

\section{References}

Abd el-Fattah, A. A., al-Yousef, H. M., al-Bekairi, A. M., \& alSawaf, H. A. (1998). Vitamin E protects the brain against oxia dative injury stimulated by excessive aluminum intake. Biochemistry and Molecular Biology International, 46(6), 1175-1180.

Alamed, J., Chaiyasit, W., McClements, D., J., \& Decker, E. A (2009). Relationships between free radical scavenging and antioxidant activity in foods. Journal of Agricultural and Food Chemistry, 57(7), 2969-2976

Alvira, D., Yeste-Velasco, M., Folch, J., Verdaguer, E., Canudas, A. M., Pallàs, M., et al. (2007). Comparative analysis of the effects of resveratrol in two apoptotic models: inhibition of complex I and potassium deprivation in cerebellar neurons. Neuroscience, 147(3), 746-756.

Ataie, A., Sabet-Kasaei, M., Haghparast, A., Moghaddam, A H., \& Kazemi-Nejad, B. (2010). Neuroprotective effects of the polyphenolic antioxidant agent, Curcumin, against homocysteine-induced cognitive impairment and oxidative stress in the rat. Pharmacology Biochemistry E Behaviour, 96(4), 378-385.

Atwood, C. S., Moir, R. D., Huang, X, Scarpa, R. C., Bacarra, N. M., Romano, D. M., et al. (1998). Dramatic aggregation of Alzg heimer abeta by $\mathrm{Cu}$ (II) is induced by conditions representing physiological acidosis. Journal of Biological Chemistry, 273(21), 12817-12826.

Bastianetto, S., Yao, Z. X., Papadopoulos, V., \& Quirion, R. (2006). Neuroprotective effects of green and black teas and their catechin gallate esters against betaamyloid-induced toxicity. European Journal of Neuroscience, 23(1), 55-64.

Baum, L., \& Ng, A. (2004). Curcumin interaction with copper and iron suggests one possible mechanism of action in Alzheimer's disease animal models. Journal of Alzheimer's Disease, $6(4), 367-377$

Behl, C., Davis, J. B., Lesley, R., \& Schubert, D. (1994). Hydrogen peroxide mediates amyloid beta protein toxicity. Cell, 77(6), 817-827.

Bertelli, A. A., \& Das, D. K. (2009). Grapes, wines, resveratrol, and heart health. Journal of Cardiovascular Pharmacology, 54(6), 468-476.

Berr, C., Balansard, B., Arnaud, J., Roussel A. M., \& Alpérovitch, A. (2000). Cognitive decline is associated with systemic oxidative stress: The EVA study. Etude du Vieillissement Arteriel. Journal of the American Geriatrics Society, 48(10), 1285-1291.

Christen, Y. (2000). Oxidative stress and Alzheimer's disease. American Journal of Clinical Nutrition, 71(2), 621S-629S.

Devore, E. E., Grodstein, F., van Rooij, F. J., Hofman, A., Stampfer, M. J., \& Witteman, J. C., et al. (2010). Dietary antioxidants and long-term risk of dementia. Archives of Neurology, 67(7): 819-825.

Dutta, K., Ghosh, D., \& Basu, A. (2009). Curcumin protects neuronal cells from Japanese encephalitis virus-mediated cell death and also inhibits infective viral particle formation by dysregulation of ubiquitin-proteasome system. Journal of Neuroimmune Pharmacology, 4(3), 328-337.

Ehrnhoefer, D., E., Duennwald, M., Markovic, P, Wacker, J. L. Engemann, S., Roark, M., et al. (2006). Green tea (-)-epigallocatechin-gallate modulates early events in huntingtin misfold- 
ing and reducestoxicity in Huntington's disease models. $\mathrm{Hu}$ man Molecular Genetics, 15(18), 2743-2751.

Galano, A., \& Francisco-Marquez, M. (2009). Peroxyl-radicalscavenging activity of garlic: 2-propenesulfenic acid versus allicin. Journal Physical Chemistry B, 113(49), 16077-16081.

Ganguli, M., Chandra, V., Kamboh, M. I., Johnston, J. M., Dodge, H. H., Thelma, B. K., et al. (2000). Apolipoprotein E polymorphism and Alzheimer's disease: The Indo-US Cross-National Dementia Study. Archives of Neurology, 57(6), 824-830.

Hardy, J. A., \& Higgins, G. A. (1992). Alzheimer's disease: the amyloid cascade hypothesis. Science, 256(5054), 184-185.

Jang, M., Cai, L., \& Udeani, G., O., Slowing, K. V., Thomas, C. F., Beecher, C. W., Fong, H. H., et al. (1997). Cancer chemopreventive activity of resveratrol, a natural product derived from grapes. Science, 275(5297), 218-220.

Karlsson, J., Emgard, M., Brundin, P., \& Burkitt, M. J. (2000) Trans-resveratrol protects embryonic mesencephalic cells from tert-butyl hydroperoxide: electron paramagnetic resonance spin trapping evidence for a radical scavenging mechanism. Journal of Neurochemistry, 75(1), 141-150.

Khan, M., M., Ahmad, A., Ishrat, T., Khan, M. B., Hoda, M. N. Khuwaja, G., et al. (2010). Resveratrol attenuates 6-hydroxydopamine-induced oxidative damage and dopamine depletion in rat model of Parkinson's disease. Brain Research, 1328, 139-151.

Kelsey, N. A., Wilkins, H. M., \& Linseman, D. A. (2010). Nutraceutical Antioxidants as Novel Neuroprotective Agents. Molecules, 15(11), 7792-7814.

Kim, H., Park, B., S., Lee, K., G., Choi, C. Y., Jang, S. S., Kim, Y. $\mathrm{H}$., et al. (2005). Effects of naturally occurring compounds on fibril formation and oxidative stress of beta-amyloid. Journal of Agricultural and Food Chemistry, 53(22), 8537-8541.

Kim, D., Nguyen, M. D., Dobbin M. M., Fischer, A., Sananbenesi, F., Rodgers, J. T., et al. (2007). SIRT1 deacetylase protects against neurodegeneration in models for Alzheimer's disease and amyotrophic lateral sclerosis. EMBO Journal, 26(13), 31693179 .

Koh, S. H., Kwon, H., Kim, K. S., Kim, J., Kim, M. H., Yu, H J., et al. (2004). Epigallocatechin gallate prevents oxidativestress-induced death of mutant $\mathrm{Cu} / \mathrm{Zn}$-superoxide dismutase (G93A) motoneuron cells by alteration of cell survival and death signals. Toxicology, 202(3), 213-225.

Launer, L. J. (2005). The epidemiologic study of dementia: a lifelong quest? Neurobiology of Aging, 26(3), 335-340.

Levites, Y., Amit, T., Youdim, M., B., \& Mandel, S. (2002). Involvement of protein kinase Cactivation and cell survival/ cell cycle genes in green tea polyphenol (-)- epigallocatechin 3-gallate neuroprotective action. Journal of Biological Chemistry, 277(34), 30574-30580

Levites, Y., Weinreb, O., Maor, G., Youdim, M. B., \& Mandel, S. (2001). Green tea polyphenol (-)-epigallocatechin-3-gall late prevents N-methyl-4-phenyl-1,2,3,6-etrahydropyridineinduced dopaminergic neurodegeneration. Journal of Neurochemistry, 78(5), 1073-1082.

Li, Y., Zhao, S., Zhang, W., Zhao, P., He, B., Wu, N., et al. (2011). Epigallocatechin-3-O-gallate (EGCG) attenuates FFAs-induced peripheral insulin resistance through AMPK pathway and insulin signalling pathway in vivo. Diabetes Research and Clinical Practice, 93(2), 205-214.

Lim, G. P., Chu, T., Yang, F., Beech, W., Frautschy, S. A., \& Cole, G. M. (2001). The curry spice curcumin reduces oxidative damage and amyloid pathology in an Alzheimer's transgenic mouse. Journal of Neuroscience, 21(21), 8370-77

Marambaud, P., Zhao, H., \& Davies, P. (2005). Resveratrol promotes clearance of Alzheimer's disease amyloid-beta peptides. Journal of Biological Chemistry, 280(45), 37377-37382.

Masaki, K. H., Losonczy, K. G., Izmirlian, G., Foley, D. J., Ross, G. W., Petrovitch, H., et al. (2000). Association of vitamin E and $\mathrm{C}$ supplement use with cognitive function and dementia in elderly men. Neurology, 54(6), 1265-1272.

Mason, R. P., Leeds, P. R., Jacob, R. F., Hough, C. J., Zhang, K. G., Mason P. E., et al. (1999). Inhibition of excessive neuronal apoptosis by the calcium antagonist amlodipine and antioxidants in cerebellar granule cells. Journal of Neurochemistry, 72(4), 1448-1456

Miloso, M., Bertelli, A. A., Nicolini, G., \& Tredici, G. (1999). Resveratrol-induced activation of the mitogen-activated protein kinases, ERK1 and ERK2, in human neuroblastoma SH-SY5Y cells. Neuroscience Letters, 264(1-3), 141-144.

Miura, K., Kikuzaki, H., \& Nakatani, N. (2002). Antioxidant activity of chemical components from sage (Salvia officinalis L.) and thyme (Thymus vulgaris L.) measured by the oil stability index method. Journal of Agricultural and Food Chemistry, 50(7), 1845-1851.

Morris, M. C., Evans, D. A., Bienias, J. L., Tangney, C. C., Bennett, D. A., Aggarwal N., et al. (2002). Dietary intake of antioxidant nutrients and the risk of incident Alzheimer's disease in a biracial community study. Journal of the American Medical Association, 287(24), 3230-3237.

Morel, I., Lescoat, G., Cogrel, P., Sergent, O., Pasdeloup, N., Brissot, P., et al. (1993). Antioxidant and iron-chelating activities of the flavonoids catechin, quercetin and diosmetin on ironloaded rat hepatocyte cultures. Biochemical Pharmacology, 45(1), 13-19.

Nunomura, A., Perry, G., Pappolla, M. A., Wade, R., Hirai, K., Chiba, S., et al. (1999). RNA oxidation is a prominent feature of vulnerable neurons in Alzheimer's disease. Journal of Neuroscience, 19(6), 1959-1964.

Pan, T., Fei, J., Zhou, X., Jankovic, J., \& Le, W. (2003). Effects of green tea polyphenols on dopamine uptake and on MPP+-induced dopamine neuron injury. Life Sciences, 72(29), 1073-1083.

Paquay, J. B., Haenen, G. R., Stender, G., Wiseman, S. A., Tijburg, L. B., \& Bast, A. (2000). Protection against nitric oxide toxicity by tea. Journal of Agricultural and Food Chemistry, 48(11), 5768-5772.

Qi, X. (2010). Reactive oxygen species scavenging activities and inhibition on DNA oxidative damage of dimeric compounds from the oxidation of (-)-epigallocatechin-3-O-gallate. Fitoterapia, 81(3), 205-209.

Rajeswari, A. (2006). Curcumin protects mouse brain from oxidative stress caused by 1-methyl-4- phenyl-1,2,3,6-tetrahydropyridine. European Review for Medical and Pharmacological Sciences, 10(4), 157-161.

Ramassamy, C., \& Poirier, J. (2001). Ginkgo biloba extract (EGb 761) and apolipoprotein E isoforms on the ß-amyloid fibril for- 
mation. Ginkgo Biloba Extract (EGb 761) and Neurodegenerative Diseases, 71-91.

Sano, M., Ernesto, C., Thomas, R. G., Klauber, M. R., Schafer, K., Grundman, M., et al. (1997). A controlled trial of selegiline, alpha-tocopherol, or both as treatment for Alzheimer's disease. The Alzheimer's Disease Cooperative Study. The New England Journal of Medicine, 336(17), 1216-1222.

Schroeder, E. K., Kelsey, N. A., Doyle, J., Breed, E., Bouchard, R. J., Loucks, F. A., et al. (2009). Green tea epigallocatechin 3-gallate accumulates in mitochondria and displays a selective antiapoptotic effect against inducers of mitochondrial oxidative stress in neurons. Antioxidants and Redox Signalling, 11(3), 469-480.

Shang, Y. J., Qian, Y. P., Liu, X. D., Dai, F., Shang, X. L., Jia, W Q., et al. (2009). Radical-scavenging activity and mechanism of resveratrol-oriented analogues: Influence of the solvent, radical, and substitution. Journal of Organic Chemistry, 74(14), 5025-5031.

Smith, J. V., \& Luo, Y. (2003). Elevation of oxidative free radicals in Alzheimer's disease models can be attenuated by Ginkgo biloba extract EGb 761. Journal of Alzheimer's Disease, 5(4), 287-300.

Sun, A., Cheng, J. (1999). Novel targets for therapeutic intervention against ischemic brain injury. Clinical Neuropharmacology, 22(3), 164-171.

Sun, A. Y., Wang, Q., Simonyi, A., \& Sun, G. Y. (2008). Botanical phenolics and brain health. NeuroMolecular Medicine, 10(4), 259-274.

Tagami, M., Yamagata, K., Ikeda, K., Nara, Y., Fujino, H. Kubota, A., et al. (1998). Vitamin E prevents apoptosis in cortical neurons during hypoxia and oxygen reperfusion. Laboratory Investigation, 78(11), 1415-1429.

Zamin, L. L., Dillenburg-Pilla, P., Argenta-Comiran, R., Horn, A. P., Simão, F., Nassif, M., et al. (2006). Protective effect of res) veratrol against oxygen-glucose deprivation in organotypic hippocampal slice cultures: Involvement of PI3-K pathway. Neurobiology of Disease, 24(1), 170-82.

Yang, C., Zhang, X., Fan, H., \& Liu, Y. (2009). Curcumin upregulates transcription factor Nrf2, HO-1 expression and protects rat brains against focal ischemia. Brain Research, 1282, 133-141.

Wu, W. M. (2005). An investigation into the neuroprotective effects of Estrogen and Progesterone in a model of Homocysteine-induced neurodegeneration (Master's Thesis). South Africa: Rhodes University. 
